\title{
BM] Global Health A double-edged sword - telemedicine for maternal care during COVID-19: findings from a global mixed-methods study of healthcare providers
}

\author{
Anna Galle (D , ${ }^{1}$ Aline Semaan, ${ }^{2}$ Elise Huysmans, ${ }^{2}$ Constance Audet, ${ }^{2}$ \\ Anteneh Asefa (D) ,2 Therese Delvaux, ${ }^{3}$ Bosede Bukola Afolabi (D) , ${ }^{4}$ \\ Alison Marie El Ayadi (D) , ${ }^{5}$ Lenka Benova ${ }^{2}$
}

To cite: Galle A, Semaan A, Huysmans $\mathrm{E}$, et al. A doubleedged sword-telemedicine for maternal care during COVID-19: findings from a global mixedmethods study of healthcare providers. BMJ Global Health 2021;6:e004575. doi:10.1136/ bmjgh-2020-004575

Handling editor Seye Abimbola

- Additional material is published online only. To view, please visit the journal online (http://dx.doi.org/10.1136/ bmjgh-2020-004575).

Received 27 November 2020 Revised 3 February 2021 Accepted 10 February 2021

Check for updates

\section{(c) Author(s) (or their} employer(s)) 2021. Re-use permitted under CC BY-NC. No commercial re-use. See rights and permissions. Published by BMJ.

For numbered affiliations see end of article.

Correspondence to Dr Anna Galle; anna.galle@ugent.be

\section{ABSTRACT}

Introduction The COVID-19 pandemic has led to a rapid implementation of telemedicine for the provision of maternal and newborn healthcare. The objective of this study was to document the experiences with providing telemedicine for maternal and newborn healthcare during the pandemic among healthcare professionals globally. Methods The second round of a global online survey of maternal and newborn health professionals was conducted, disseminated in 11 languages. Data were collected between 5 July and 10 September 2020. The questionnaire included questions regarding background, preparedness and response to COVID-19, and experiences with providing telemedicine. Descriptive statistics and qualitative thematic analysis were used to analyse responses, disaggregated by country income level. Results Responses from 1060 maternal and newborn health professionals were analysed. Telemedicine was used by $58 \%$ of health professionals and two-fifths of them reported not receiving guidelines on the provision of telemedicine. Key telemedicine practices included online birth preparedness classes, antenatal and postnatal care by video/phone, a COVID-19 helpline and online psychosocial counselling. Challenges reported lack of infrastructure and technological literacy, limited monitoring, financial and language barriers, lack of nonverbal feedback and bonding, and distrust from patients. Telemedicine was considered as an important alternative to in-person consultations. However, health providers emphasised the lower quality of care and risk of increasing the already existing inequalities in access to healthcare. Conclusions Telemedicine has been applied globally to address disruptions of care provision during the COVID-19 pandemic. However, some crucial aspects of maternal and newborn healthcare seem difficult to deliver by telemedicine. More research regarding the effectiveness, efficacy and quality of telemedicine for maternal healthcare in different contexts is needed before considering longterm adaptations in provision of care away from faceto-face interactions. Clear guidelines for care provision and approaches to minimising socioeconomic and technological inequalities in access to care are urgently needed.

\section{Key questions}

What is already known?

- Telemedicine is the delivery of healthcare services by healthcare professionals from distance through using information and communication technologies for the exchange of valid and correct information.

- Telemedicine for maternal and newborn health can safely be used to deliver certain components of care in highly controlled settings where the technology is available and accessible to patients.

- Telemedicine has been applied rapidly and on a wide scale during the COVID-19 pandemic to replace face-to-face visits along the continuum of maternal and newborn healthcare.

\section{What are the new findings?}

- Maternal and newborn healthcare providers globally considered telemedicine of benefit during the pandemic and applied it on a wide scale for different aspects of maternal and newborn healthcare.

- The rapid adaptation to provision of care via telemedicine was not optimally supported by guidelines training for health providers, adequate equipment, reimbursement for cost of connectivity and insurance payments for care provided remotely.

- Healthcare providers worldwide reported not being able to reach a substantial group of families by telemedicine and encountered different barriers in providing high-quality maternity care by telemedicine, and such challenges were more prominent in lowincome and middle-income countries.

\section{INTRODUCTION}

The WHO declared COVID-19 a pandemic on 11 March 2020, as a consequence of the more than 118000 cases spread over 110 countries and the sustained risk of further global spread. ${ }^{1}$ The overall response strategy in many countries for fighting the pandemic included early diagnosis, patient isolation, monitoring of contacts, isolation of suspected 


\section{Key questions}

What do the new findings imply?

- Pre-existing inequalities in access to high-quality care might have increased by the large scale and rapid implementation of telemedicine during the COVID-19 pandemic in different settings.

- Uptake of telemedicine by women was hampered by various factors such as internet connection problems, lack of the necessary equipment, digital illiteracy, and distrust.

- In-depth research is needed to formalise evidence-based guidelines for the implementation of telemedicine along the continuum of maternal and newborn care, as lessons learnt for building back beyond the COVID-19 pandemic and also for future emergency preparedness.

and confirmed cases, and some extent of lockdown. ${ }^{2}$ In this context, the vital role of telemedicine, video consultations in particular, rapidly increased in order to reduce the risk of transmission, especially in settings where insufficient personal protective equipment was available for the health workforce. The call for implementing telehealth rapidly has never been louder, especially in high-income countries (HICs) where technological resources are widely available..$^{3-5}$ As a consequence, an updated framework for telemedicine in the COVID-19 pandemic for aiding national governments in defining their strategy against COVID-19 was proposed at the start of the pandemic, advising telehealth for all routine care and (suspected) COVID-19 positive cases with mild symptoms. ${ }^{4}$ This framework and other variations have been used on a large scale by governments and health systems globally to define and implement their public health response to the COVID-19 outbreak while maintaining the provision of essential health services and avoiding the so-called 'COVID-19 collateral'. ${ }^{467}$

According to the WHO, telehealth is the delivery of healthcare services by healthcare professionals from distance through using information and communication technologies for the exchange of valid and correct information. ${ }^{8}$ Sometimes the term telemedicine is specifically used to refer to service delivery by physicians only, while telehealth includes service provision by nurses, pharmacists and other health professionals. In a broader definition, the term telehealth also includes the interactions between healthcare providers (eg, delivery of training, team meetings) and the interaction of a patient with technology in the absence of a health provider (eg, an automated phone line, a health application (app) on a mobile phone).$^{9}$ In this paper, we use the terms telemedicine and telehealth synonymously and interchangeably. ${ }^{8}$ A wide variety of telehealth interventions exists today, including the use of mobile phone apps, online health education modules, web portals, wearable devices, text messaging (SMS) and live audio-visual communication. ${ }^{9}$ The benefits of investing in telemedicine (sometimes also referred to as m-health or e-health ${ }^{10-12}$ ) have been discussed in the literature mainly from a public health perspective, documenting the many successes in different health service domains and countries, ${ }^{13-15}$ ranging from economic efficiency to overcoming distance barriers in remote areas. ${ }^{16}$ Telemedicine applications seem most commonly used and evaluated for the management of chronic diseases and mental disorders in HICs. ${ }^{17-20}$ Recently, the use of telehealth has expanded dramatically during the COVID-19 pandemic due to the necessity of maintaining service provision while ensuring physical distancing as a strategy for slowing down the transmission of the virus, and limited accessibility of healthcare to both patients and providers. ${ }^{3}$

Previous epidemic outbreaks and events disruptive to health systems showed the potential of telemedicine in avoiding further spread of an epidemic disease and maintaining some provision of general healthcare. ${ }^{4}$ A mobile app named Ebola Contact Tracing could successfully monitor and trace contacts of confirmed cases during the Ebola virus outbreak in Sierra Leone and healthcare providers were educated and trained via a virtual tutorial. ${ }^{2122}$ Studies also explored the use of telehealth in the context of natural disasters and conflicts, with promising results. In the aftermath of two hurricanes in the USA in 2017, telemedicine was successfully used for the implementation of free two-way video consultations for victims, although the lack of infrastructure and Wi-Fi access were cited as serious challenges. ${ }^{23}$

Since the start of the COVID-19 pandemic, telehealth has been increasingly implemented in many countries and different health domains to maintain health service provision to some extent. ${ }^{24}$ Several studies have reported positive results in terms of providers' and patients' experiences, and clinical outcomes, ${ }^{25}{ }^{26}$ but the evidence base so far is limited and covers a narrow range of health services and contexts. For handling confirmed COVID-19 cases with mild symptoms, the maximum use of telemedicine for guaranteeing providers' and other patients' safety has not been questioned as an essential part of reducing the spread of the virus. ${ }^{27} 28$ On the other hand, the necessity for and evidence regarding providing some elements of care for healthy pregnant women and newborns through telemedicine remain scarce. ${ }^{29}$

The evidence on telehealth interventions in maternal and newborn healthcare before the COVID-19 pandemic is mixed. Two recent systematic reviews found that telehealth interventions were associated with improvements in obstetric outcomes, perinatal smoking cessation, continuation of breast feeding, monitoring of highrisk pregnancies and early access to medical abortion services. ${ }^{30}$ The included studies presented a wide variety of telehealth interventions in maternal healthcare, including use of mobile phone apps, wearable devices, SMS and live audio-visual communication. However, only studies conducted in HICs and China, a rapidly growing middle-income country (MIC), were included in both reviews. Robust evidence from low/middle-income countries (LMICs) is lacking, mainly due to poor methodical quality of studies and their narrow scope (often focusing on a single application in a specific setting) ${ }^{32} \mathrm{SMS}$ support 
for women during pregnancy seemed the most promising and commonly evaluated telehealth intervention in low-income country (LIC) contexts, and was associated with increased utilisation of healthcare, early initiation of breast feeding, uptake of recommended prenatal and postnatal care (PNC) consultations, skilled birth attendance and infant vaccination. ${ }^{10} 153233$ Telehealth applications for maternal health have been implemented also in conflict settings and among migrant women before the COVID-19 pandemic, where they appear useful for providing maternal health education. ${ }^{34-36}$

The importance of telehealth for maintaining the provision and use of essential maternal and newborn health services during the COVID-19 pandemic has been highlighted by the $\mathrm{WHO} .^{29}$ In its operational guidance for the COVID-19 context, the WHO identified health interventions during the antenatal, intrapartum and postnatal periods as essential services, and suggested the use of telemedicine when the technology is available. ${ }^{29}$ Evidence regarding its implementation, effectiveness, feasibility, cost-effectiveness and health outcomes is beginning to emerge but remains scarce. Available reports, mostly commentaries and grey literature, have highlighted some challenges and concerns for implementing telemedicine in maternal and newborn health alongside its advantages. The availability of technology and connectivity seem to pose a serious bottleneck, together with high start-up costs and lack of health insurance reimbursement for care provided remotely. ${ }^{37}$ Concerns regarding a 'digital divide', meaning increasing maternal and newborn health disparities and inequities as a consequence of access to technology and connectivity, have also been raised. ${ }^{39} 40$

Despite the efforts to supplement the reduction in the provision of in-person maternal and newborn care by telemedicine during COVID-19, little is known about the actual implementation of these efforts and barriers to their effectiveness as perceived by healthcare providers. This paper documents the findings of a rapid online global survey of maternal and newborn health professionals during the COVID-19 pandemic, focusing on their experience of providing care to pregnant and postpartum women and their newborns using telemedicine.

\section{METHODS}

\section{Study design}

We present the findings from the second round of a repeated cross-sectional online survey of maternal and newborn healthcare providers. We focus on the application of telemedicine for maintaining the provision of maternal and newborn healthcare during the COVID-19 outbreak. The survey targeted midwives, nurses, obstetricians/gynaecologists, neonatologists and other health professionals. An invitation to complete the survey was distributed to those who responded to the first round of the survey, and to other healthcare providers through personal networks of the multicountry research team members, maternal/newborn platforms and social media (eg, Facebook, Twitter, WhatsApp groups). Additional details about the study design, sampling and findings of the first round of the survey were published previously. ${ }^{41}$

\section{Questionnaire}

A team of international collaborators adapted the questionnaire used in the first round of the survey in light of the evolving situation of the pandemic. The team included health professionals and experts in health systems, maternal health epidemiologists and public health researchers, acknowledged in a previously published commentary ${ }^{42}$ and paper based on the first round of the global survey. ${ }^{41}$ The core structure of the first survey round was maintained and we collected data on respondents' background, preparedness for COVID19, response to COVID-19 and own work experience during the pandemic. We additionally aimed to expand our understanding and explore some of the themes that were developed during analysis of the responses received during the first round more in depth. ${ }^{43}$ We added a section on the use of telemedicine, where we asked participants whether they used technology to counsel or provide care to women or their babies remotely, and if so, which services. We asked whether they received any guidelines on telemedicine provision. Finally, we also asked whether they used telemedicine in the same way compared with before the pandemic, more, or only started since the beginning of the pandemic.

In open text responses, we asked respondents to share the top three successes and challenges that they experienced using telemedicine. Further, respondents could share their general concerns about providing care during the pandemic in an open text box at the end of the questionnaire. The questionnaire was available in 11 languages (English, French, Arabic, Italian, Portuguese, Spanish, Japanese, German, Dutch, Russian and Kiswahili). The questionnaire is available publicly ${ }^{44}$ and the questions relevant to telemedicine are provided in online supplemental file 1 .

\section{Data processing and analysis}

We included responses collected between 5 July 2020 and 10 September 2020. We cleaned the 1331 responses received by removing duplicate submissions $(n=14)$, refusals to participate $(\mathrm{n}=131)$, submissions with more than $85 \%$ of questions with missing answers $(n=46)$ and submissions from respondents who skipped all the telemedicine questions $(n=80)$. Quantitative and qualitative analyses were done simultaneously in a concurrent design. Quantitative analysis involved producing descriptive statistics (frequencies and percentages) using Stata/SE V.14. Descriptive statistics revealed the overrepresentation of healthcare providers from Kazakhstan in our sample. This was a result of a proactive dissemination of the survey by the Ministry of Health in Kazakhstan. A sensitivity analysis was conducted, showing that in Kazakhstan $67 \%$ of respondents used telemedicine vs 
$52 \%$ from other MICs. We did not apply any statistical corrections for the sampling because this mixed-methods analysis did not aim to make generalisable statements about telemedicine use by country income levels. The Kazakhstani responses were taken into consideration when summarising and interpreting the qualitative data.

All open-ended text responses were translated to English by AG (fluent in Spanish, Portuguese, English, Dutch and French) and by AS (Arabic), with additional assistance from the research team with translating and interpreting responses received in other languages. Responses to open-ended questions were analysed using Braun and Clarke's six-phase framework for thematic analysis and inductive coding. ${ }^{43}$ This framework involves a reflexive process of moving forward (and sometimes backward) through data familiarisation, coding, theme development, revision, naming and writing up. The open-ended responses were read and reread in order to generate initial ideas. Data were then systematically coded by one researcher (AG), and the developed codes and themes were discussed on a weekly basis with the multidisciplinary coauthor team (including a midwife, nurse, medical doctor, anthropologist, maternal health epidemiologist and quantitative public health scientist). Inconsistent codes were rejected or adapted and overarching themes were developed. The last two phases involved refining the themes extracted from the data, adding quotes and double checking if the themes really reflected the respondents' experiences and perceptions with feedback from the coauthor group. Throughout this process, we paid special attention to the context in which the participants' experiences and thoughts were rooted (ie, country, position in the team, cadre). Finally, the continuum of maternal and newborn care was used as a framework for visualising the results. ${ }^{45}$

\section{Patient and public involvement}

No patient or public involvement took place in the design or conduct of this study. We involved health professionals, experts in health systems, infectious diseases, infection prevention and control, and health epidemiologists, and public health researchers from various global settings in the design of this study and the survey tool. We intend to disseminate the main results to several stakeholders and health professionals globally by social media and personal contacts, including to the participants of the study.

\section{RESULTS}

Table 1 displays the background characteristics of the 1060 survey participants included in the analysis. Respondents worked in 71 different countries, most commonly in Kazakhstan $(n=507)$, the Democratic Republic of the Congo $(n=43)$, Italy $(n=43)$, Nigeria $(n=37)$ and Japan $(n=34)$. The most common cadres of health professionals in the sample were nurses (29\%), midwives and nursemidwives (25\%), and obstetricians/gynaecologists (21\%). The majority of respondents identified as women (78\%).
Table 1 Respondents' background and workplace characteristics $\left(n=1060^{*}\right)$

n (\%)

\begin{tabular}{|c|c|}
\hline \multicolumn{2}{|l|}{ Country income level† } \\
\hline High-income & $277(26)$ \\
\hline Middle-income & $682(64)$ \\
\hline Low-income & $101(10)$ \\
\hline \multicolumn{2}{|l|}{ World region } \\
\hline East Asia and Pacific & $41(4)$ \\
\hline Europe and Central Asia & $675(64)$ \\
\hline Latin America and Caribbean & $66(6)$ \\
\hline Middle East and North Africa & $53(5)$ \\
\hline North America & $25(2)$ \\
\hline South Asia & $27(2)$ \\
\hline Sub-Saharan Africa & $173(16)$ \\
\hline \multicolumn{2}{|l|}{ Cadre } \\
\hline Midwife/nurse-midwife & $257(25)$ \\
\hline Nurse & $312(29)$ \\
\hline Obstetrician/gynaecologist & $223(21)$ \\
\hline Neonatologist/paediatrician & $73(7)$ \\
\hline Medical doctor (no specialisation) & $126(12)$ \\
\hline Other & $54(5)$ \\
\hline \multicolumn{2}{|l|}{ Position } \\
\hline Head of facility & $44(4)$ \\
\hline Head of department or ward & $103(10)$ \\
\hline Head of team & $87(8)$ \\
\hline Team member & $237(22)$ \\
\hline Interim member & $74(7)$ \\
\hline Independent or self-practicing & $110(10)$ \\
\hline Other & $360(34)$ \\
\hline \multicolumn{2}{|l|}{ Gender } \\
\hline Female & $826(78)$ \\
\hline Male & $213(20)$ \\
\hline Prefer not to mention & $7(2)$ \\
\hline
\end{tabular}

Type of care provided (multiple responses allowed)

\begin{tabular}{lc} 
Outpatient ANC & $402(38)$ \\
Home-based childbirth care & $76(7)$ \\
Outpatient PNC & $321(30)$ \\
Outpatient breastfeeding support & $255(24)$ \\
Inpatient ANC & $284(27)$ \\
Inpatient childbirth care & $362(34)$ \\
Inpatient PNC & $325(31)$ \\
Surgical care & $169(16)$ \\
Neonatal care (small and sick newborns) & $157(15)$ \\
Home visits & $152(14)$ \\
Community outreach & $204(19)$ \\
Family planning provision or counselling & $251(24)$ \\
Abortion care & $139(13)$ \\
Post-abortion care & $189(18)$ \\
\hline
\end{tabular}

Continued 
Table 1 Continued

\begin{tabular}{|c|c|}
\hline & n (\%) \\
\hline Other & $147(14)$ \\
\hline \multicolumn{2}{|l|}{ Works in more than one health facility } \\
\hline Yes & $742(70)$ \\
\hline No & $300(28)$ \\
\hline \multicolumn{2}{|l|}{ Primary workplace } \\
\hline Referral hospital & $202(19)$ \\
\hline District/regional hospital & $196(18)$ \\
\hline Health centre & $91(8)$ \\
\hline Polyclinic or clinic & $280(26)$ \\
\hline Birth centre & $111(10)$ \\
\hline Independent or self-practicing & $67(6)$ \\
\hline Other & $94(9)$ \\
\hline \multicolumn{2}{|l|}{ Primary workplace sector } \\
\hline Public (national) & $532(50)$ \\
\hline Public (university or teaching) & $107(10)$ \\
\hline Public (district level or below) & $168(16)$ \\
\hline Private for profit & $28(3)$ \\
\hline Private not-for-profit & $25(3)$ \\
\hline Independent or self-practicing & $63(6)$ \\
\hline Other & $88(8)$ \\
\hline \multicolumn{2}{|l|}{ Type of area } \\
\hline Large city (>1 million inhabitants) & $355(33)$ \\
\hline Small city (100000-1 million inhabitants) & $246(23)$ \\
\hline Town (<100000 inhabitants) & $149(14)$ \\
\hline Village/rural area & $236(22)$ \\
\hline Other & $43(4)$ \\
\hline \multicolumn{2}{|l|}{ Workplace characteristics } \\
\hline Facility provides caesarean section & $535(50)$ \\
\hline Facility accepts referrals from other facilities & $672(63)$ \\
\hline
\end{tabular}

${ }^{*}$ Differential number of missing values across variables. †According to the World Bank classification. ${ }^{84}$

ANC, antenatal care; PNC, postnatal care.

Respondents were involved in providing outpatient antenatal care (ANC) $(38 \%)$, outpatient PNC (30\%), inpatient childbirth care $(34 \%)$ and inpatient PNC $(31 \%)$. Three-quarters of respondents worked in public health facilities, almost half worked in urban settings (large and small cities), and 22\% worked in villages or rural areas.

Among the entire sample, $58 \%$ of health professionals reported using some form of telemedicine (figure 1). This includes all those who used telemedicine more than before the pandemic, in the same way as before the pandemic, and those who started using telemedicine since the beginning of the pandemic. Three-quarters of respondents from LICs were not using telemedicine at all at the time of their response, compared with $41 \%$ of those working in HICs and 24\% in MICs. The percentage of healthcare providers introducing telemedicine since the beginning of the pandemic was higher among those who worked in HICs and MICs $(18 \%$ and $16 \%$, respectively) compared with LICs (1\%).

Among the 612 respondents who provided healthcare using telemedicine, $65 \%$ used telemedicine to provide routine ANC, 59\% used it to provide childbirth preparation sessions, half provided routine $\mathrm{PNC}$ and breastfeeding counselling, $40 \%$ provided family planning counselling and $17 \%$ abortion care. Thirty-nine per cent of respondents using telemedicine reported that they did not receive guidelines on this mode of care provision.

The qualitative analysis focused on the main practices and challenges for implementing telemedicine during COVID-19 among maternal healthcare providers worldwide. We identified elements of care along the continuum of maternal and newborn healthcare which were commonly provided using telemedicine (figure 2). Those were further classified into different telemedicine practices within five broad categories: (1) education and counselling by telemedicine, (2) reducing or eliminating personal visits, (3) replacing in-person consultations by telemedicine, (4) setting up hotlines or information lines, and (5) providers connecting to one another by telehealth. The practices are explained more in detail in table 2, including the perceived benefits by providers and rationale for using them during the pandemic. Furthermore, we found eight general themes (not related to a specific component of care or practice) concerning the challenges encountered when providing telemedicine: lack of infrastructure, technological illiteracy, remote monitoring limitations, financial barriers, lack of nonverbal feedback, limited bonding, language barriers and distrust (figure 2).

\section{Practices}

The application of telemedicine along the five broad categories was slightly different according to the context, but they seemed to commonly exist in every world region. Healthcare providers using telemedicine were in general positive and enthusiastic about its potential for continuing healthcare provision during the pandemic without a transmission risk. For example, a midwife from Norway commented that 'telehealth is a brilliant way to have a look and give advice without being in touch.'

Providing online group birth preparedness classes during pregnancy was one of the most popular newly introduced telehealth practices mentioned by respondents. They explained that telemedicine was a good alternative to face-to-face classes because education and counselling is an element of care which does not involve a physical examination. This care can be delivered easily by Zoom or other video-conferencing platform/mobile app, as explained by a midwife from Costa Rica: 'It has been possible to continue childbirth preparation courses through teams or zoom. Furthermore most of the users have smartphones that allow them to access platforms to receive additional information afterwards.' Respondents who mentioned using online group birth preparedness classes mostly worked in HICs or MICs, indicating that 


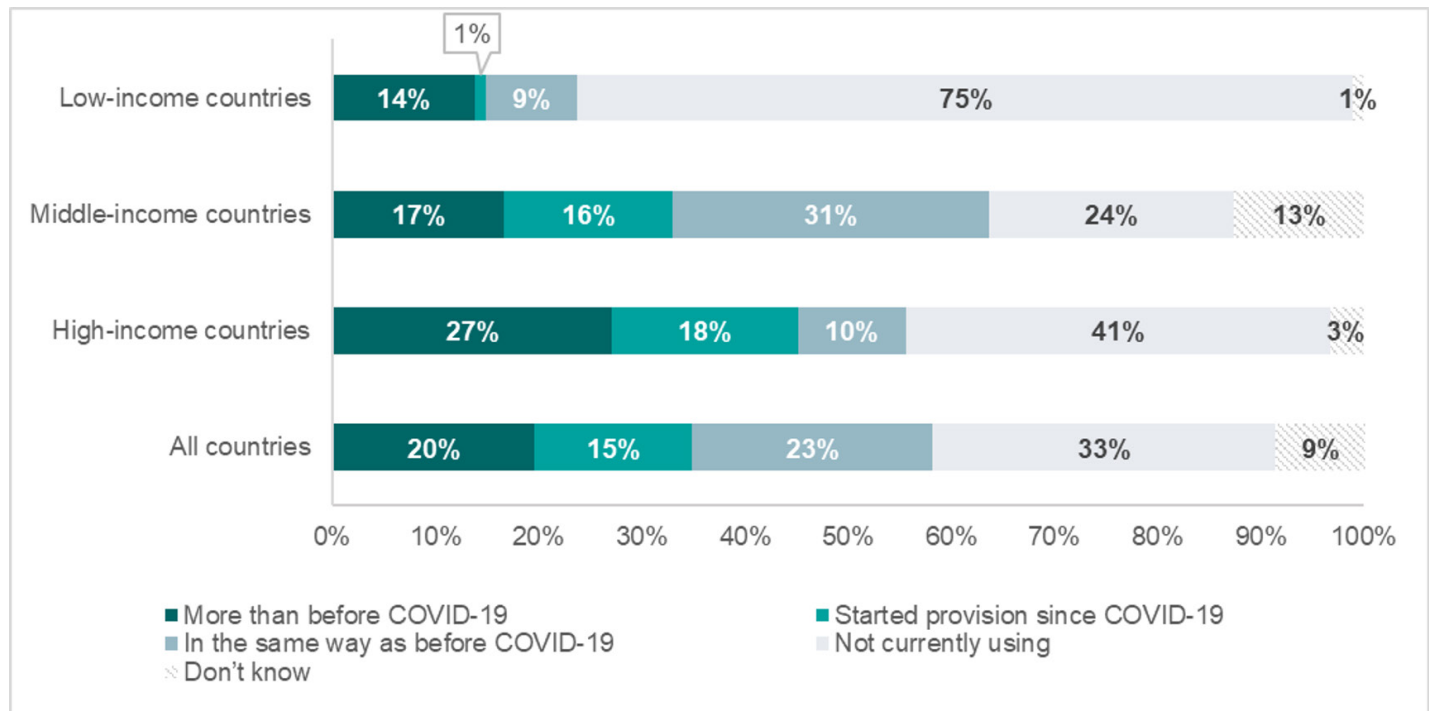

Figure 1 Percentage of respondents currently using technology to counsel or provide care to women or their babies remotely as compared with before the COVID-19 pandemic, by country income level $(\%, n=1060)$.

this switch to remote technologies in the provision of health education, in particular group sessions, might have been less common and/or accessible in LICs. Noteworthy, formally organised group classes are less common in general in some regions (eg, in many African countries health information is provided to women before/ after the start of the regular ANC consultations in health facilities, rather than specially organised birth preparedness classes ${ }^{46}$ ), which might also explain why online group classes during the pandemic were not a commonly reported practice in those settings.

The second telehealth practice concerned the reduction or elimination of non-essential personal consultations. Providers did not seem to have a standard definition of what they considered as 'essential' versus 'non-essential' health consultations. Healthcare providers used email and/or WhatsApp to send prescriptions and laboratory results to their patients if needed. This did not seem to be a completely new practice, but was more commonly performed since the beginning of the pandemic (as women and their families were discouraged from visiting health facilities unless absolutely necessary) and easily accepted by patients and implemented by providers. Medical abortion was an example of a service mentioned within this theme. A doctor from Cameroon described how he assisted women with an abortion, by providing a medical prescription and a cervical-ripening agent remotely, to reduce the time spent with the patient in person: 'One of the biggest successes of telemedicine is preparing women with abortion remotely for surgical aspiration. I come to perform it when ready.' He mentioned this telehealth practice was routine before the pandemic.

Third, providers commonly reported the application of telemedicine to replace face-to-face consultations. Practices that fall under this theme include conducting antenatal and postnatal consultations through video/phone calls (mostly WhatsApp) instead of in-person visits in the health facility or at the woman's home. Contrary to the exchange of prescriptions and lab results, this seemed

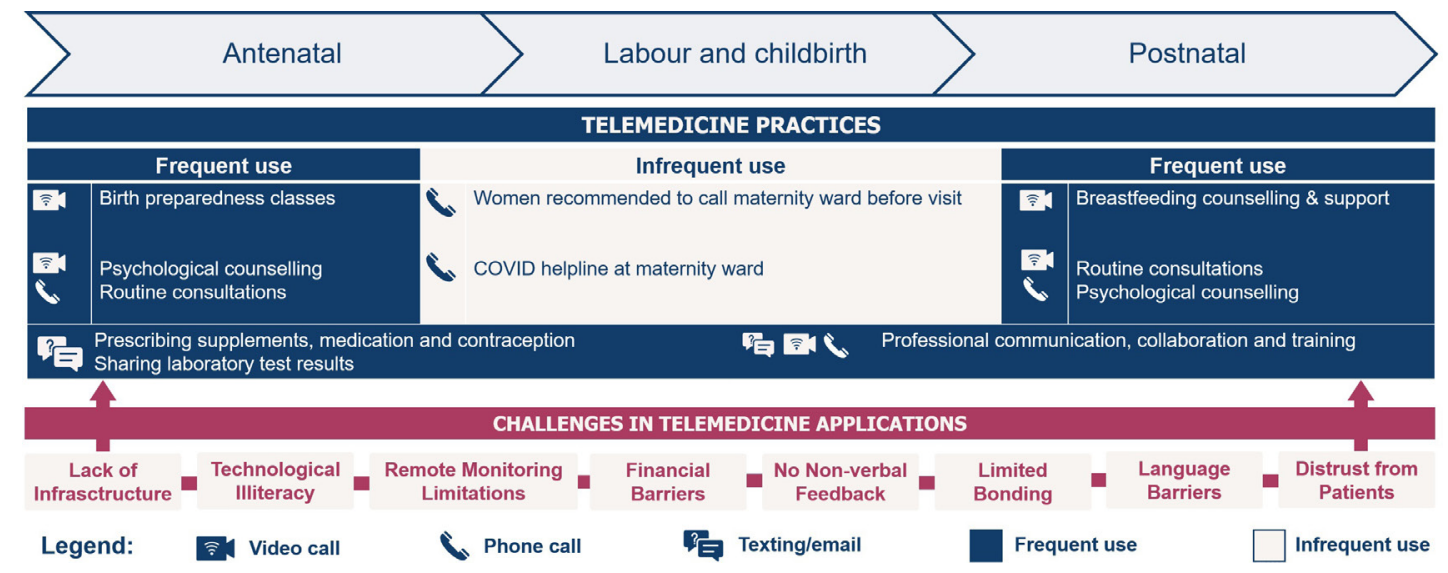

Figure 2 Key types of practices and challenges of providing care through telemedicine along the continuum of maternal and newborn healthcare $(n=612)$ users of telemedicine during COVID-19 pandemic. 
Table 2 Commonly reported uses of telemedicine in maternal healthcare related to the COVID-19 pandemic and providers' insights and perceived benefits

\begin{tabular}{|c|c|c|}
\hline Practices & Use of telemedicine & Provider's insights and perceived benefits \\
\hline \multirow{2}{*}{$\begin{array}{l}\text { Education and counselling of women } \\
\text { and their families }\end{array}$} & $\begin{array}{l}\text { Postnatal (breastfeeding) counselling and } \\
\text { support by video }\end{array}$ & $\begin{array}{l}\text { During the COVID-19 pandemic women often } \\
\text { had less access to support from family and } \\
\text { friends, making postnatal support by healthcare } \\
\text { providers (in particular, midwives) even more } \\
\text { crucial. }\end{array}$ \\
\hline & Psychosocial counselling by phone/video & $\begin{array}{l}\text { Useful to address the generally increased } \\
\text { levels of anxiety during the COVID-19 pandemic. } \\
\text { Effective to provide information and highly } \\
\text { demanded by women and their families } \\
\text { (especially regarding COVID-19 risks and } \\
\text { potential negative consequences for mother and } \\
\text { baby if infected). }\end{array}$ \\
\hline $\begin{array}{l}\text { Reducing or eliminating personal } \\
\text { visits }\end{array}$ & $\begin{array}{l}\text { Prescriptions (contraception, medication, } \\
\text { medical abortion pill) by WhatsApp/email } \\
\text { Lab results exchange by WhatsApp/email }\end{array}$ & $\begin{array}{l}\text { Visits to the facility can be avoided by giving } \\
\text { prescriptions and/or medications for longer } \\
\text { periods than usual. } \\
\text { Face-to-face consultation time can be } \\
\text { reduced or eliminated when people receive } \\
\text { digital information/prescriptions/lab results. }\end{array}$ \\
\hline $\begin{array}{l}\text { Replacing in-person consultations } \\
\text { with telemedicine }\end{array}$ & $\begin{array}{l}\text { Antenatal and postnatal care consultations by } \\
\text { phone/video }\end{array}$ & $\begin{array}{l}\text { More often used if pregnancies were low risk, } \\
\text { while high risk often continued with in-person } \\
\text { visits. } \\
\text { Often a personal decision by the health } \\
\text { provider whether to replace in-person visits by } \\
\text { telehealth or not, because of the lack of official } \\
\text { guidelines. } \\
\text { Easy to schedule a convenient time for } \\
\text { woman and health provider. }\end{array}$ \\
\hline
\end{tabular}

an application of telemedicine newly introduced since the beginning of the COVID-19 pandemic. Respondents and patients in some countries were not able to access health facilities due to strict curfews, lockdowns, public transport bans and closures of health facilities, making face-to-face consultation almost completely impossible. Telehealth was described as a solid alternative to compensate the lack of face-to-face consultations to some extent, although with limitations. A nurse-midwife from Uganda commented that 'through phone calls to postnatal mothers on breastfeeding, cord care, and thermal care, babies have survived although they were not able to access the health facilities for postnatal care.' The practice of remote blood pressure or fetal heartbeat monitoring by women themselves (self-monitoring) was not reported by respondents, indicating that this was not a common practice in our sample. Importantly, respondents reported cancelling some antenatal and postnatal consultations without replacement by any form of telehealth, especially for pregnancies considered low risk. Currently, the WHO recommends eight ANC contacts for a low-risk pregnancy. ${ }^{48}$ Some providers reported reverting to a reduced number of ANC visits by using the pre-2016 WHO recommendation of four focused ANC visits. ${ }^{49}$ A midwife from Kenya explained that conducting fewer ANC consultations was done in order to reduce the 
risk of infection during an ANC visit: 'Overall, we have less consultations because we give women less appointments so that we reduce the risk of being contaminated during consultation.'

Respondents reported that care during labour and delivery was provided in person during the COVID-19 pandemic. Women continued to be advised to give birth in health facilities. Nevertheless, many healthcare providers reported a decrease in the number of facility-based births during the pandemic. The only new applications of telehealth for intrapartum care during the pandemic were that women in labour were requested to make a phone call before travelling to the hospital. In addition, in circumstances of shortened hospital stays after childbirth (in some cases just a couple of hours after birth up to a maximum of 48 hours for a normal delivery), the first days of postpartum care were almost entirely delivered by outpatient care, which was frequently done by telehealth. Some respondents mentioned that only the 6 -week postpartum visit was retained as an in-person visit, because it coincides with the newborn's vaccination schedule. Respondents also reported that women were more in need of guidance and support during the postpartum period (for example with breast feeding), in part also due to reduced informal support by friends and family.

The fourth telehealth practice was established as healthcare providers tried to respond to what they described as higher levels of anxiety, psychosocial problems, questions and insecurity among pregnant and postpartum women, all instigated by the pandemic. Women wanted more information about the effects of a COVID-19 infection on pregnancy and newborn, as well as information about COVID-19-related restrictions and procedures in the hospital. Healthcare workers reported using online consultations, phone calls and text messages to provide this information and support. Some health facilities also temporarily installed telephone hotlines to answer patients' questions. Telehealth seemed to grant healthcare providers, midwives in particular, a feeling of connectedness with and care for their patients under the difficult circumstances of COVID-19. A midwife from Bangladesh described how telehealth combated loneliness and even saved lives: 'By providing telehealth first of all women are not completely alone. Besides that, it also has saved lives because I advised women by the phone to come on time for the delivery and provided remote abortion care.'

Fifth, beyond the application of telemedicine as a means of contact between patients and providers, we found that many providers reported a positive effect of the pandemic on collaboration between health facilities and among healthcare providers. They reported an increase in the use of telecommunication for exchange of information and expertise both among colleagues and at institutional levels. A midwife from Germany explained how team work improved as a consequence: 'One of the successes during COVID in my organization were the more frequent team meetings, partially done online, which enabled uniform action against the spread of the virus.' Healthcare providers also mentioned improved interdisciplinary collaboration by sharing guidelines (mostly clinical COVID-19 protocols) and updates by WhatsApp/ email/phone calls. The restrictions regarding physical training courses also increased providers' participation in online training modules and simulations.

\section{Challenges}

Among the healthcare providers providing telemedicine $(n=612)$, almost half $(n=282,46 \%)$ reported challenges with this mode of service provision in open text responses. The decision to offer telehealth often seemed a personal decision made by health providers, based on a risk-benefit assessment. Eight broad categories of challenges were identified: lack of infrastructure, technological illiteracy, remote monitoring limitations, financial barriers, lack of non-verbal feedback, limited bonding, language barriers and distrust. Among respondents using telemedicine in the same way as before the pandemic only the first two challenges were reported. Among respondents introducing telemedicine during the pandemic or using it more than before, all eight challenges were reported.

\section{Lack of infrastructure}

Most healthcare providers seemed to use their own smartphone for providing telehealth services and one of the biggest challenges reported was poor internet connection and/or regular interruptions in connectivity. This was a global problem reported by providers from both LMICs and HICs. As noted by a midwife in the USA: 'Trying to connect with women from rural areas with poor wi-fi service was a challenge.' In LICs, electricity cuts were also mentioned by several respondents, affecting their ability to provide telehealth, as described by a midwife in Nepal: 'The electricity cuts are bothering the most. Besides, internet fall outs.'

\section{Technological illiteracy}

Lack of skills to manage the software and devices for conducting telehealth seemed a major obstacle for both women and health providers. While people might have access to the necessary equipment (such as a smartphone), they were reluctant to use it for telehealth because they do not know how to handle the technology correctly. Installing and using the necessary app on a mobile phone or program on the computer seemed the most difficult step for many people, although it was difficult to understand at which point problems emerged from providers' reports. A midwife from France noted that: 'Women do not always understand how to use the technology properly.' Also at the provider's side a midwife from Argentina mentioned a lack of technological skills: 'Many providers do not use telemedicine because learning the skills to do it are a personal responsibility instead of an institutional responsibility.'

\section{Remote monitoring limitations}

Healthcare providers reported that their inability to perform physical examinations (such as fetal heartbeat monitoring, blood pressure measurement or assessing fundal height) was one of the most important shortcomings of antenatal, 
postnatal and neonatal consultations through video/phone calls. A neonatologist in Japan who was providing care for women and their newborns after discharge described the most important challenges as follows: 'When I do an online consultation I cannot do any direct examination, I cannot observe any reaction to an examination and I cannot do any medical scan.'

\section{Financial barriers}

While many providers were very enthusiastic about the use of telemedicine, they also noted that it is not affordable for many of their patients because they lack the financial means to purchase the necessary technical devices or airtime (also called talk-time or data allowance). WhatsApp (installed on a smartphone) was mentioned as communication medium most often and also preferred by healthcare providers, but it became clear that not everyone had a smartphone at their disposal. This was a recurring theme in both LMICs and HICs. A medical doctor in India wrote that 'the use of the phone, SMS and WhatsApp is a success for telemedicine but only $30 \%$ of the people have a smartphone.'

Respondents themselves faced financial burdens from the use of telemedicine on two levels: not being able to afford the equipment and lack of reimbursement. Several mentioned the absence of reimbursement for costs they incurred while providing telemedicine (including the telehealth consultation itself and its associated internet/phone/data costs). Respondents from LICs particularly reported that there was no standard way of getting reimbursed for providing telehealth consultations. Many patients did not have insurance or insurance companies did not cover the telemedicine consultations. Providers did not know how to invoice the telemedicine consultations as patients were not coming personally anymore. In addition, particularly midwives often mentioned the burden of having to pay the internet/phone costs from their own pocket; this was perceived as a serious additional barrier. These financial issues were affecting healthcare providers' willingness and ability to provide care, as noted by a midwife in Kenya in regard to the cost of mobile data: 'Sometimes I cannot do a follow up of the patients because of lack of airtime.'

\section{Lack of non-verbal feedback}

Many healthcare providers reported they could not be assured that the health information was well understood by women because they could not read their facial and/or body expressions. Midwives reported this problem more frequently than obstetricians. A midwife in Nigeria elaborated: 'I don't like offering remote care. I feel that if you are not seeing the non-verbal cues and facial expression of your patient, you will not truly know if they are ok.'

\section{Limited relationship and bonding}

Closely related to the lack of non-verbal feedback, also the relationship and bond between the midwife and a woman was affected by telemedicine. This was described by a midwife in the USA as follows: 'Technology is a good tool, but does not replace face-to-face conversations, palpating a mom's abdomen, and listening to the baby's heart rate in order to form warm, trusting bonds between a patient and the midwife.' This theme was typical among midwives who felt that the personal interaction was part of 'being a midwife': 'My main concern is that we are not at the bedside as midwives traditionally are', noted a midwife in the USA.

\section{Language barriers}

Language barriers were perceived to be more problematic during telemedicine compared with in-person consultations. Healthcare providers found it easier to overcome language barriers during in-person visits by using body language, which was not feasible virtually. Also, the use of interpreters was sometimes not possible or more problematic for online consultations compared with face-to-face visits. A nursemidwife from the USA described how both language barriers and financial barriers hampered access to telehealth: 'Using medical interpreters over the video is a real challenge. Furthermore, our most disadvantaged patients also have limited access to telephone or video.'

\section{Distrust}

Healthcare providers perceived that some patients had little trust in the care provided through telemedicine and were reluctant to accept it. One specific problem perceived by health providers was that undocumented migrants refused telehealth consultations because they were afraid to be recorded during such interactions and feared a possible prosecution. A midwife in the USA explained: 'Many of my patients are not documented or are in the U.S. temporarily, and are thus reluctant to participate in video and telephone visits due to well-grounded fears of information being recorded or listened to by government agencies.'

\section{DISCUSSION}

This study used a rapid global online survey to understand the care process adaptations used by more than a thousand maternal and newborn health providers from over 70 countries during the COVID-19 pandemic. We found that telemedicine was frequently used for various services along the continuum of maternal and newborn healthcare and differed somewhat across contexts. The choice of providing telemedicine was often a personal decision of each health professional, rather than a health facility policy, or a country guideline. This means that some healthcare providers reported that personal financial costs were a serious barrier to provision. Telemedicine was already practised to a certain extent before the pandemic by two-fifths of the respondents, but more widely implemented during the pandemic by one in five. Some healthcare providers also introduced telemedicine for the first time during the pandemic. Health providers implementing telemedicine for the first time or using it more often during the pandemic faced more challenges than those using it to the same extent as before. Globally, maternal and newborn health providers in LICs seem to face more severe barriers to implement telehealth practices compared with those in middle-income and high-income settings. 
In this paper, we show that maternal and newborn health professionals adapted the provision of care using telemedicine during the COVID-19 pandemic in many different ways, even within similar settings. The lack of evidencebased consolidated national or global guidelines together with a legal framework on the usage of telehealth might explain these findings. ${ }^{9}{ }^{16}$ Among healthcare providers who were using telemedicine, two-fifths reported that they did not receive any guideline on the provision of care through telemedicine. Currently, guidelines on telemedicine mostly originate from national medical specialty societies outside maternal healthcare provision and are not tailored to the context of a pandemic, limiting their transferability to other health domains and contexts. ${ }^{9}$ Furthermore, health systems and governments did not seem to be prepared for the rapid evolution of the pandemic, and maintaining the provision of maternal and newborn healthcare might not have been their first priority. Only after the first pandemic peak in March/ April 2020 did the first reports of a potential disruptive effect of the pandemic on maternal and newborn care provision start to emerge,${ }^{750-53}$ allowing more coordinated action from stakeholders and governments.

Our study showed that several factors played a role in the decision of healthcare providers on whether to implement telehealth during COVID-19, for which care and to which patients. These factors included a risk-benefit assessment, personal preference of the provider and patient, financial consequences and health status of the women (low-risk vs high-risk pregnancies). Some healthcare providers declared that they only saw women with a high-risk pregnancy and shifted entirely to telemedicine for low-risk women. Other providers and facilities shifted to the previous recommendation of four focused ANC visits for low-risk pregnancies, ${ }^{48}$ replacing some of the remaining visits with telemedicine consultations or a hotline in case of emergencies and questions. A recent publication from India described a similar approach here advising face-to-face ANC provision for high-risk pregnant women and a reduced number of visits for low-risk women, although they also claimed that more robust data are needed to evaluate the effectiveness of their approach. ${ }^{54}$ Obstetric care is characterised by unpredictability; women may develop complications throughout their pregnancy, even when they were classified as low risk. ${ }^{55}$ As a consequence, a low-risk profile might change to high risk rapidly without warning. Such changes in risk status may go unnoticed, given that healthcare providers reported that providing care via telehealth risks losing certain essential information (related to non-verbal feedback and physical examinations), which they believed might affect the quality of care. Previous evidence showed that receiving fewer ANC consultations than the recommended has a negative effect on maternal and newborn health outcomes. ${ }^{56-58}$ Unfortunately, evidence-based guidelines about the ideal number of telehealth visits versus face-to-face visits during pregnancy and post partum are lacking, together with guidelines on the integration of home-based equipment (eg, blood pressure monitor, glucometer, urine analysis test strips) which could provide important information to increase care quality and detection of possible complications. In-depth research is needed to assist healthcare providers with guidance on how to implement telehealth along the continuum of maternal and newborn healthcare, and ensure the provision of high-quality maternal health services through blended approaches.

Our study showed that many providers experienced serious challenges in organising and conducting teleconsultations. The most important challenges when providing telehealth included lack of infrastructure, technological illiteracy, financial barriers, remote monitoring limitations, lack of non-verbal feedback, limited bonding, language problems and distrust from patients. Lack of infrastructure varied from internet connection problems to a lack of smartphones and/ or other devices at one or both sides of the clinical encounter (providers and patients). While the lack of internet and equipment was most often reported in LICs, ${ }^{59}$ it appeared to be a global problem according to our data and also common among health providers being familiar with telemedicine (ie, those using it already before the pandemic). Our study showed that a large proportion of healthcare providers and patients do not have easy and affordable access to telemedicine equipment and mobile/data networks. Furthermore, recent research has revealed a gender gap in mobile internet use in LMICs with women being $20 \%$ less likely to use mobile internet than men. ${ }^{60}$ Given that maternal health is primarily directed to women and that majority of maternal healthcare workers are women, this gender gap will negatively affect the use of telehealth for maternal healthcare. If telehealth is intended to fill the gaps of healthcare provision during periods of disruptions to healthcare supply and utilisation, or even an essential part of the general healthcare system, this access issue will need to be addressed, possibly through government subsidies or grants for the most disadvantaged groups. ${ }^{61}$

Technological illiteracy was another problem commonly reported in our study by respondents from all types of countries. Offering education programmes, investing in userfriendly software and social outreach programmes might all be strategies to reduce technological illiteracy and the hidden digital inequality and health disparities ${ }^{62}$ that might emerge with wider telemedicine use. Financial issues in using telehealth seemed most problematic in LICs for both providers and patients, according to our study. A big share of women cannot afford to buy airtime for consultations and do not have access to (smart) phones. Healthcare providers also reported struggling to afford equipment and airtime. In addition, especially healthcare providers from LMICs found it difficult to get paid for telehealth consultations due to a lockdown and/or lack of reimbursement system (by a health institution or social security system). In contrast, health providers in HICs often had access to phones and internet offered by their institutions. Furthermore, health insurance companies are increasingly covering the main part of the telehealth consultations in line with regular care in several HICs, which makes the financial compensation for telehealth less problematic for them. ${ }^{63-65}$ It is noteworthy that rapidly evolving technological solutions are coming up in both LICs 
and HICs (for example, by allowing money transfer by SMS or WhatsApp ${ }^{66}$ ), which might overcome certain barriers for providing telehealth in the future.

In our study, telemedicine was mostly performed by conducting video consultations without reports of patients self-monitoring of vital signs, fetal heartbeat or glucose levels. Providers reported this as a challenge (described as 'remote monitoring limitations') and serious risk for guaranteeing high-quality care. Indeed, very little is known about the benefits and risks of introducing this simplified method and scope of telehealth for providing maternal and newborn healthcare worldwide. Currently, evaluation studies or randomised controlled trials are hardly available within the literature regarding this new way of healthcare provision, and more research is definitely needed to discover its impact on women and newborns' health outcomes.

Distrust, language problems, lack of non-verbal feedback and bonding were reported as barriers to telemedicine in our study. A study in Bangladesh reporting very similar barriers with telemedicine explained this by emphasising that a provider's physical presence can easily express empathy and compassion non-verbally, while this is much more difficult during a telehealth consultation. ${ }^{68} \mathrm{~A}$ similar concern was reported by midwives in our study who revealed they could not build a warm and trusting bond with the women by telehealth consultations.

Healthcare providers who introduced telemedicine during the pandemic or used it more often faced more serious barriers compared with those using telemedicine in the same way as before. Most of the reported challenges in this study seem related to the rapid scale-up of telemedicine, whereby face-to-face consultations were almost completely impossible due to several COVID-19-related restrictions. We assume health providers using telemedicine to the same extent as before the pandemic faced fewer challenges, allowing a more balanced and natural way of telemedicine provision. The latest evidence from other health domains shows that digital modes of communication work best for patients and clinicians who have already previously established relationships, with a flexible use of telecommunication according to patient condition and background. ${ }^{18}$ We believe that also in maternal healthcare a more balanced and natural combination of face-to-face and telemedicine contacts between women and health providers, under more flexible circumstances, might improve the experience of women and healthcare providers with telemedicine consultations in the future.

Contrary to the challenges of telemedicine, the benefits of telehealth applications in maternal healthcare are well documented. Many studies show that shifting to telemedicine for certain aspects of care is equally beneficial as face-to-face care when it comes to health outcomes and patient satisfaction. ${ }^{69-72}$ However, it is important to note that these studies mainly derive from the USA and are conducted in a highly controlled setting with adequate equipment for remote monitoring of blood pressure and blood glucose levels. ${ }^{69} 7072$ One study in Japan also reported successful telemedicine provision during COVID-19 by documenting their remote ANC consultation procedure that included the mailing of a cardiotocograph and a sphygmomanometer to each pregnant woman's home for remote monitoring. ${ }^{73}$ Unfortunately, these telehealth interventions do not correspond with the global practice of telehealth during the pandemic, where it has been applied in very different ways and under suboptimal circumstances. Furthermore, telehealth is an already dynamic and rapidly evolving field, resulting in additional challenges for in-depth monitoring and evaluation of new applications. $^{16}$

Our study suggests that vulnerable groups are at risk of being excluded from telemedicine, perhaps even higher than from routine in-person maternal healthcare. Given that groups such as single women, adolescents, migrants and women of low socioeconomic status already face challenges in reaching face-to-face maternal healthcare services, ${ }^{74-76}$ it is crucial that shifting to telehealth does not exacerbate these inequalities. It is important to note that even with concrete guidelines regarding the implementation of telehealth, a one-size-fits-all model will not be appropriate. Each country must continually assess which groups of society are vulnerable to exclusion and fairly support those at the highest risk. ${ }^{77}$

Our study showed that in general, providers appreciated the application of telemedicine and that it was often the only way to 'connect' with women, families and their newborns. Midwives reported higher levels of loneliness and depression among both pregnant and postpartum women, which is in line with the first studies in the field of maternal mental health during the COVID-19 era and previous epidemic outbreaks. ${ }^{78-80}$ On one hand the virtual meetings, counselling and support by midwives can help women, but on the other they are only partially doing 'the job' because of the lack of physical contact and bonding. More research is needed on how the mental health needs in the perinatal period can be addressed by telehealth during a pandemic or similarly disruptive situations. ${ }^{81} 82$

Lastly, the COVID-19 pandemic seemed to boost the communication and interdisciplinary management of care between healthcare providers by mobile technologies. Our study showed that the increased ability to reach colleagues and specialists for advice was valuable to healthcare providers during the pandemic, besides receiving up-to-date guidelines by virtual communication. Furthermore, they explained it could avoid unnecessary referrals between hospitals by soliciting advice from experts by phone. A Cochrane review also showed that mobile health communication between providers probably decreases the time to deliver healthcare, as well as the number of face-to-face appointments, ${ }^{83}$ both essential aspects of the global strategy to reduce the spread of the COVID-19 virus. It is critical that this improved collaboration and communication will be continued after the pandemic, as the benefits and lessons learnt will be important to tackling long-standing issues such as communication during the referral process.

\section{Limitations}

Limitations of our study are the sample bias and lack of representativeness, due to the convenience sampling approach. We received few responses from professionals working in 
lower level facilities, particularly in LICs, which might itself be related to limited access to the internet in these settings. Our sample might over-represent higher qualified cadres of health professionals in settings with limited use of technology among lower cadres of staff, and under-represent overwhelmed providers, and those with limited access to internet connection. This is particularly relevant for the topic of this analysis, as we might overestimate the use of telehealth because of the sample that we reached. Another limitation of this study is the cross-sectional design, whereby we only captured the experiences of providers at one given moment. While we could grasp from the responses which telemedicine practices were applied more often or introduced during the pandemic, it was not always clear to determine to what extent these practices were used before the pandemic. Follow-up research, using a longitudinal design, could determine to what extent certain telehealth applications continue and evolve over time and after the pandemic, assessing their perceived benefits and challenges for maternal healthcare provision in the long term. Lastly, the findings related to the positive effect of the crisis on professional telecommunication and collaboration were presented briefly and grouped together as one telehealth practice because they were not the focus of our study. Nevertheless, they are equally important, especially for improving maternal health outcomes and health providers' work satisfaction. We believe further research is needed with regard to this particular aspect of telehealth, to support long-term improvements in collaboration and multidisciplinary teamwork among health providers.

\section{CONCLUSION}

Maternal and newborn health providers considered telehealth to be an important alternative to providing certain health services during the first months of the COVID-19 pandemic. It gave them the possibility to connect with patients and interact with other health professionals without being exposed to the risks of in-person contact, or when facing restrictions to movement. Furthermore, telehealth seems to be less time-consuming (sometimes with equal financial compensation) and can easily be combined with other duties at home or in health facilities. However, more research is needed to understand the consequences of an extensive telehealth consultation schedule in maternal and newborn health during the COVID-19 pandemic. Some authors already pointed to the risk of loneliness and depression for women giving birth during the pandemic, where the lack of interpersonal contact during the postpartum period and increased stress levels seemed serious triggers. We believe the negative consequences might go beyond that, taking into account the reduced number of in-person ANC visits and digital inequality that goes hand in hand with providing telehealth. Illiterate, migrant, poor and ethnic minority women appear to be particularly left behind in accessing maternal health by telehealth. More research regarding the effectiveness and efficacy of telehealth for maternal healthcare in different contexts is highly needed before implementing such adaptations in the long term and on a large scale, particularly to avoid an increase in the existing wide inequalities in access to maternal healthcare worldwide.

\section{Author affiliations}

${ }^{1}$ ICRH, Department of Public Health and Primary Care, Ghent University, Gent, Belgium

${ }^{2}$ Department of Public Health, Institute of Tropical Medicine, Antwerpen, Belgium

${ }^{3}$ Public Health, Institute of Tropical Medicine, Antwerpen, Belgium

${ }^{4}$ Department of Obstetrics and Gynaecology, University of Lagos, Idi-Araba, Nigeria

${ }^{5}$ Obstetrics, Gynecology and Reproductive Sciences, University of California, San

Francisco, California, USA

Twitter Anteneh Asefa @AntenehAsef, Bosede Bukola Afolabi @Coolgynae and Lenka Benova @lenkabenova

Acknowledgements We would like to thank the study participants who took time to respond to this survey during the second round, despite the ongoing difficult circumstances and high workload. We acknowledge the Institutional Review Committee at the Institute of Tropical Medicine for providing helpful suggestions on this study protocol, and for the expedited review of this study. We thank all study collaborators, translators and colleagues who distributed the invitation for this survey and provided suggestions on the questionnaire, including the coauthors of this paper.

Contributors LB conceptualised the study and obtained funding. All authors contributed to the design of the study and development of the survey tool. AG analysed the qualitative data and $A S$ analysed the quantitative data. AG, AS and LB wrote the original draft of the manuscript. All authors contributed to the development of the manuscript, and read and approved the final version.

Funding This study was funded by the Institute of Tropical Medicine's COVID-19 Pump Priming fund supported by the Flemish Government, Science and Innovation. LB is funded in part by the Research Foundation-Flanders (FWO) as part of her senior postdoctoral fellowship.

\section{Competing interests None declared.}

\section{Patient consent for publication Not required.}

Ethics approval This study was approved by the Institutional Review Committee at the Institute of Tropical Medicine (Antwerp, Belgium) on 20 March 2020 (approval reference 1372/20). Respondents provided informed consent online by checking a box affirming that they voluntarily agreed to participate in the survey.

Provenance and peer review Not commissioned; externally peer reviewed.

Data availability statement Data are available upon request. Anonymised data analysed during the current study will be made available from the corresponding author upon reasonable request.

Supplemental material This content has been supplied by the author(s). It has not been vetted by BMJ Publishing Group Limited (BMJ) and may not have been peer-reviewed. Any opinions or recommendations discussed are solely those of the author(s) and are not endorsed by BMJ. BMJ disclaims all liability and responsibility arising from any reliance placed on the content. Where the content includes any translated material, BMJ does not warrant the accuracy and reliability of the translations (including but not limited to local regulations, clinical guidelines, terminology, drug names and drug dosages), and is not responsible for any error and/or omissions arising from translation and adaptation or otherwise.

Open access This is an open access article distributed in accordance with the Creative Commons Attribution Non Commercial (CC BY-NC 4.0) license, which permits others to distribute, remix, adapt, build upon this work non-commercially, and license their derivative works on different terms, provided the original work is properly cited, appropriate credit is given, any changes made indicated, and the use is non-commercial. See: http://creativecommons.org/licenses/by-nc/4.0/.

\section{ORCID iDs}

Anna Galle http://orcid.org/0000-0003-1427-5067

Anteneh Asefa http://orcid.org/0000-0003-4470-1848

Bosede Bukola Afolabi http://orcid.org/0000-0002-7511-7567

Alison Marie El Ayadi http://orcid.org/0000-0003-2674-4887

\section{REFERENCES}

1 WHO. WHO Director-General's opening remarks at the media briefing on COVID-19 - 11 March 2020. Available: https://www. 
who.int/director-general/speeches/detail/who-director-general-sopening-remarks-at-the-media-briefing-on-covid-19---11-march2020 [Accessed 13 Oct 2020].

2 Haug N, Geyrhofer L, Londei A. Ranking the effectiveness of worldwide COVID-19 government interventions. medRxiv 2020.

3 Smith AC, Thomas E, Snoswell CL, et al. Telehealth for global emergencies: implications for coronavirus disease 2019 (COVID-19). $J$ Telemed Telecare 2020;26:309-13.

4 Ohannessian R, Duong TA, Odone A. Global telemedicine implementation and integration within health systems to fight the COVID-19 pandemic: a call to action. JMIR Public Health Surveill 2020;6:e18810.

5 Bashshur R, Doarn CR, Frenk JM, et al. Telemedicine and the COVID-19 pandemic, lessons for the future. Telemed J E Health 2020;26:571-3.

6 Bokolo Anthony. Use of telemedicine and virtual care for remote treatment in response to COVID-19 pandemic. J Med Syst 2020;44:132.

7 Graham WJ, Afolabi B, Benova L, et al. Protecting hard-won gains for mothers and newborns in low-income and middle-income countries in the face of COVID-19: call for a service safety net. BMJ Glob Health 2020;5:e002754.

8 WHO Global Observatory for eHealth. Telemedicine: opportunities and developments in member states: report on the second global survey on eHealth. World Health organization; 2010. https://apps. who.int/iris/handle/10665/44497

9 Tuckson RV, Edmunds M, Hodgkins ML. Telehealth. N Engl J Med 2017;377:1585-92.

10 WHO. Monitoring and evaluating digital health interventions, 2016. Available: https://www.who.int/reproductivehealth/publications/ mhealth/digital-health-interventions/en/\#.X50Simip8k8.mendeley [Accessed 31 Oct 2020].

11 Ngidi WH, Naidoo JR, Ncama BP, et al. Mapping evidence of interventions and strategies to bridge the gap in the implementation of the prevention of mother-to-child transmission of HIV programme policy in sub-Saharan countries: a scoping review. Afr J Prim Health Care Fam Med 2017;9:e1-10.

12 Nair U, Armfield NR, Chatfield MD, et al. The effectiveness of telemedicine interventions to address maternal depression: a systematic review and meta-analysis. $J$ Telemed Telecare 2018;24:639-50.

13 Monaghesh E, Hajizadeh A. The role of telehealth during COVID-19 outbreak: a systematic review based on current evidence. $B M C$ Public Health 2020;20.

14 Novara G, Checcucci E, Crestani A, et al. Telehealth in urology: a systematic review of the literature. how much can telemedicine be useful during and after the COVID-19 pandemic? Eur Urol 2020;78:786-811.

15 Mbuthia F, Reid M, Fichardt A. mHealth communication to strengthen postnatal care in rural areas: a systematic review. $B M C$ Pregnancy Childbirth 2019;19:406.

16 Chang $\mathrm{H}$. Evaluation framework for telemedicine using the logical framework approach and a fishbone diagram. Healthc Inform Res 2015;21:230-8.

17 Armfield NR, Bradford M, Bradford NK. The clinical use of SkypeFor which patients, with which problems and in which settings? A snapshot review of the literature. Int J Med Inform 2015;84:737-42.

18 Ignatowicz A, Atherton $\mathrm{H}$, Bernstein $\mathrm{CJ}$, et al. Internet videoconferencing for patient-clinician consultations in long-term conditions: a review of reviews and applications in line with guidelines and recommendations. Digit Health 2019:5:205520761984583.

19 Bashshur RL, Shannon GW, Bashshur N, et al. The empirical evidence for telemedicine interventions in mental disorders. Telemed $J$ E Health 2016;22:87-113.

20 Zhou X, Snoswell CL, Harding LE, et al. The role of telehealth in reducing the mental health burden from COVID-19. Telemed JE Health 2020;26:377-9.

21 Innovations for Poverty Action. Reducing Ebola Virus Transmission: Improving Contact Tracing in Sierra Leone. Available: http://www. poverty-action.org/study/reducing-ebola-virus-transmissionimproving-contact-tracing-sierra-leone [Accessed 20 Nov 2020].

22 Using mobile technology to improve maternal health and fight Ebola 2. Available: http://www.grameenfoundation.org/press- [Accessed 20 Nov 2020].

23 Uscher-Pines L, Fischer S, Tong I, et al. Virtual first responders: the role of direct-to-consumer telemedicine in caring for people impacted by natural disasters. J Gen Intern Med 2018;33:1242-4.

24 Mehrotra A, Ray K, Brockmeyer DM. Rapidly Converting to "Virtual Practices": Outpatient Care in the Era of Covid-19. NEJM Catalyst 2020 https://catalyst.nejm.org/doi/full/10.1056/CAT.20.0091
25 Khairat S, Pillai M, Edson B, et al. Evaluating the telehealth experience of patients with COVID-19 symptoms: recommendations on best practices. J Patient Exp 2020;7:665-72.

26 Aziz A, Zork N, Aubey JJ, et al. Telehealth for high-risk pregnancies in the setting of the COVID-19 pandemic. Am J Perinatol 2020;37:800-8.

27 Monaghesh E, Hajizadeh A. The role of telehealth during COVID-19 outbreak: a systematic review based on current evidence. BMC Public Health 2020;20:1193.

28 Greenhalgh T, Koh GCH, Car J. Covid-19: a remote assessment in primary care. BMJ 2020;368:m1182.

29 WHO. Maintaining essential health services: operational guidance for the COVID-19 context: interim guidance, 1 June 2020. Available: https://apps.who.int/iris/handle/10665/332240

30 Xie W, Dai P, Qin Y, et al. Effectiveness of telemedicine for pregnant women with gestational diabetes mellitus: an updated meta-analysis of 32 randomized controlled trials with trial sequential analysis. BMC Pregnancy Childbirth 2020;20:198.

31 DeNicola N, Grossman D, Marko K, et al. Telehealth interventions to improve obstetric and gynecologic health outcomes. Obstet Gynecol 2020;135:371-82.

32 Lee SH, Nurmatov UB, Nwaru BI, et al. Effectiveness of mHealth interventions for maternal, newborn and child health in low- and middle-income countries: systematic review and meta-analysis. $J$ Glob Health 2016;6:010401.

33 Abaza H, Marschollek M. mHealth application areas and technology Combinations*. A comparison of literature from high and low/middle income countries. Methods Inf Med 2017;56:e105-22.

34 UNRWA. An application that tracks the health of mother and child: every pregnant women's dream! Available: https://www.unrwa.org/ newsroom/features/application-tracks-health-mother-and-childevery-pregnant-women's-dream [Accessed 20 Nov 2020].

35 Improving the health care of pregnant refugee and migrant women and newborn children (2018). Available: https://www.euro.who.int/ en/publications/abstracts/improving-the-health-care-of-pregnantrefugee-and-migrant-women-and-newborn-children-2018

36 Hughson J-AP, Daly JO, Woodward-Kron R, et al. The rise of pregnancy apps and the implications for culturally and linguistically diverse women: narrative review. JMIR Mhealth Uhealth 2018;6:e189.

37 Fryer K, Delgado A, Foti T, et al. Implementation of obstetric telehealth during COVID-19 and beyond. Matern Child Health $J$ 2020;24:1104-10.

38 View of challenges in telemedicine implementation for pregnancy care during the Covid-19 pandemic. Available: http://jurnalkepera watanglobal.com/index.php/jkg/article/view/156/76 [Accessed 11 Jan 2021].

39 Hill I, Burroughs E. Maternal telehealth has expanded dramatically during the COVID-19 pandemic: equity concerns and promising approaches, 2020. Available: https://www.centerforsocialinclusion. org/our-work/what-is-racial-equity [Accessed 10 Nov 2020].

40 Blandford A, Wesson J, Amalberti R, et al. Opportunities and challenges for telehealth within, and beyond, a pandemic. Lancet Glob Health 2020;8:e1364-5.

41 Semaan A, Audet C, Huysmans E, et al. Voices from the frontline: findings from a thematic analysis of a rapid online global survey of maternal and newborn health professionals facing the COVID-19 pandemic. BMJ Glob Health 2020;5:2967.

42 Benova L, Sarkar NDP, Fasehun L-KO, et al. A call to action: documenting and sharing solutions and adaptations in sexual, reproductive, maternal and newborn health care provision during the COVID-19 pandemic. Sex Reprod Health Matters 2020;28:1838054.

43 Braun V, Clarke V. Thematic analysis. In: APA Handbook of research methods in psychology: research designs: quantitative, qualitative, neuropsychological, and biological. Washington DC: American Psychological Association, 2012: Vol 2. p57-71.

44 Preparedness and response to COVID-19: a global survey of maternal and newborn care providers round 2 questionnaire. Available: https://www.itg.be/Files/docs/MATCO/Maternal_ COVID19 Round2 Survey.pdf

45 Kerber KJ, de Graft-Johnson JE, Bhutta ZA, et al. Continuum of care for maternal, newborn, and child health: from slogan to service delivery. Lancet 2007;370:1358-69.

46 Attitude of pregnant women towards group health talk during antenatal care visit in a Nigerian City. Available: https://www. researchgate.net/publication/331715857_Attitude_of_Pregnant women_towards_Group_Health_Education_during_Antenatal_Care_ visit_in_a_Nigerian_City [Accessed 12 Jan 2021].

47 Sharma J, O'Connor M, Rima Jolivet R. Group antenatal care models in low- and middle-income countries: a systematic evidence synthesis. Reprod Health 2018;15:38. 
48 WHO. New guidelines on antenatal care for a positive pregnancy experience, 2017. Available: http://www.who.int/reproductivehealth/ news/antenatal-care/en/ [Accessed 14 Oct 2020].

49 Lincetto O, Mothebesoane-Anoh S, Gomez P. Antenatal care III. Available: https://www.who.int/pmnch/media/publications/ aonsectionlII_2.pdf

50 Roberton T, Carter ED, Chou VB, et al. Early estimates of the indirect effects of the COVID-19 pandemic on maternal and child mortality in low-income and middle-income countries: a modelling study. Lancet Glob Health 2020;8:e901-8.

51 Busch-Hallen J, Walters D, Rowe S, et al. Impact of COVID-19 on maternal and child health. Lancet Glob Health 2020;8:e1257.

52 WHO/Europe Maternal and Newborn Health. Coronavirus and pregnancy - preserving maternal health across the European region. Available: https://www.euro.who.int/en/health-topics/Life-stages/ maternal-and-newborn-health/news/news/2020/6/coronavirus-andpregnancy-preserving-maternal-health-across-the-european-region [Accessed 20 Nov 2020].

53 Coxon K, Turienzo CF, Kweekel L, et al. The impact of the coronavirus (COVID-19) pandemic on maternity care in Europe. Midwifery 2020;88:102779.

54 Bindra V. Telemedicine for Women's Health During COVID-19 Pandemic in India: A Short Commentary and Important Practice Points for Obstetricians and Gynaecologists. J Obstet Gynecol India 2020;70:279-82.

55 Danilack VA, Nunes AP, Phipps MG. Unexpected complications of low-risk pregnancies in the United States. Am J Obstet Gynecol 2015;212:809.e1-809.e6.

56 Raatikainen K, Heiskanen N, Heinonen S. Under-attending free antenatal care is associated with adverse pregnancy outcomes. BMC Public Health 2007;7:1-8.

57 Dowswell T, Carroli G, Duley L, et al. Alternative versus standard packages of antenatal care for low-risk pregnancy. Cochrane Database Syst Rev 2015;2015:CD000934.

58 Kuhnt J, Vollmer S. Antenatal care services and its implications for vital and health outcomes of children: evidence from 193 surveys in 69 low-income and middle-income countries. BMJ Open 2017;7:e017122.

59 Centre for Disease Control. Uses of telehealth during COVID-19 in low resource Non-U.S. settings, 2020. Available: https://www.cdc. gov/coronavirus/2019-ncov/global-covid-19/telehealth-covid19nonUS.html [Accessed 30 Sep 2020].

60 The mobile gender gap report 2020, 2020. Available: www.gsma com/r/gender-gap [Accessed 23 Nov 2020].

61 Oyediran KA, Makinde OA, Adelakin O. The role of telemedicine in addressing access to sexual and reproductive health services in sub-Saharan Africa during the COVID-19 pandemic. Afr J Reprod Health 2020;24:49.

62 Zhai Y. A call for addressing barriers to telemedicine: health disparities during the COVID-19 pandemic. Psychother Psychosom 2021;90:64-6

63 Health Advances Blog. The changing fortunes of telemedicine in Europe - past, present, and future beyond COVID-19. Available: https://healthadvancesblog.com/2020/05/06/the-changing-fortunesof-telemedicine-in-europe/ [Accessed 30 Oct 2020].

64 CCHP. State telehealth laws and reimbursement policies report. Available: https://www.cchpca.org/telehealth-policy/state-telehealthlaws-and-reimbursement-policies-report [Accessed 30 Oct 2020].

65 Society for maternal fetal medicine coding Committee SMFM coding white paper: interim coding guidance: coding for telemedicine and remote patient monitoring services during the COVID-19 pandemic,
2020. Available: https://s3.amazonaws.com/cdn.smfm.org/ media/2264/c4ccb382b8428d22cbcb2c5dd560633f-original.png [Accessed 10 Nov 2020].

66 Technology and Operations Management. M-Pesa: when "mobile money" revolutionizes banking in Africa. Available: https://digital. hbs.edu/platform-rctom/submission/m-pesa-when-mobile-moneyrevolutionizes-banking-in-africa/ [Accessed 20 Nov 2020].

67 WhatsApp Blog. Send payments in India with WhatsApp. Available: https://blog.whatsapp.com/send-payments-in-india-withwhatsapp/?lang=fb [Accessed 20 Nov 2020].

68 Chowdhury SR, Sunna TC, Ahmed S. Telemedicine is an important aspect of healthcare services amid COVID-19 outbreak: its barriers in Bangladesh and strategies to overcome. Int $J$ Health Plann Manage 2021;36:4-12.

69 Pflugeisen BM, McCarren C, Poore S, et al. Virtual visits: managing prenatal care with modern technology. MCN Am J Matern Child Nurs 2016;41:24-30.

70 Pflugeisen BM, Mou J. Patient satisfaction with virtual obstetric care. Matern Child Health J 2017;21:1544-51.

71 Odibo IN, Wendel PJ, Magann EF. Telemedicine in obstetrics. Clin Obstet Gynecol 2013;56:422-33.

72 DeNicola N, Grossman D, Marko K, et al. Telehealth interventions to improve obstetric and gynecologic health outcomes: a systematic review. Obstet Gynecol 2020;135:371-82.

73 Nakagawa K, Umazume T, Mayama M, et al. Feasibility and safety of urgently initiated maternal telemedicine in response to the spread of COVID-19: a 1-month report. J Obstet Gynaecol Res 2020;46:1967-71.

74 Ronsmans C, Graham WJ, Lancet Maternal Survival Series steering group. Maternal mortality: who, when, where, and why. Lancet 2006;368:1189-200.

75 Graham W, Woodd S, Byass P, et al. Diversity and divergence: the dynamic burden of poor maternal health. Lancet 2016;388:2164-75.

76 Kinney MV, Boldosser-Boesch A, McCallon B. Quality, equity, and dignity for women and babies. Lancet 2016;388:2066-8.

77 The Lancet .Redefining vulnerability in the era of COVID-19. Lancet 2020;395:1089.

$78 \mathrm{Wu}$ Y, Zhang C, Liu H, et al. Perinatal depressive and anxiety symptoms of pregnant women during the coronavirus disease 2019 outbreak in China. Am J Obstet Gynecol 2020;223:240.e1-240.e9.

79 Davenport MH, Meyer S, Meah VL, et al. Moms are not OK: COVID-19 and maternal mental health. Front. Glob. Womens Health 2020;1.

80 Dodgson JE, Tarrant M, Chee Y-O, et al. New mothers' experiences of social disruption and isolation during the severe acute respiratory syndrome outbreak in Hong Kong. Nurs Health Sci 2010;12:198-204.

81 Hermann A, Fitelson EM, Bergink V. Meeting maternal mental health needs during the COVID-19 pandemic. JAMA Psychiatry 2021;78:123.

82 Nair U, Armfield NR, Chatfield MD, et al. The effectiveness of telemedicine interventions to address maternal depression: a systematic review and meta-analysis. J Telemed Telecare 2018;24:639-50.

83 Gonçalves-Bradley DC, J Maria AR, Ricci-Cabello I, et al. Mobile technologies to support healthcare provider to healthcare provider communication and management of care. Cochrane Database Syst Rev 2020;8:CD012927.

84 World Bank. World bank country and lending groups - world bank data help desk, 2017. Available: https://datahelpdesk.worldbank. org/knowledgebase/articles/906519 [Accessed 10 Nov 2020]. 